THEORY OF DATA

\title{
Word-embedding Based Text Vectorization Using Clustering
}

V. I. Yuferev ${ }^{1}$, N. A. Razin ${ }^{2}$

DOI: $10.18255 / 1818-1015-2021-3-292-311$

${ }^{1}$ Department of Information Technologies of the Central Bank of the Russian Federation, Laboratory of innovations "Novosibirsk", 12 Neglinnaya str., Moscow 107016, Russia.

${ }^{2}$ Department of Counteraction to Unfair Practices, the Central Bank of the Russian Federation, 12 Neglinnaya str., Moscow 107016, Russia.

MSC2020: 97R40, 68T50

Research article

Full text in Russian

It is known that in the tasks of natural language processing, the representation of texts by vectors of fixed length using word-embedding models makes sense in cases where the vectorized texts are short.

The longer the texts being compared, the worse the approach works. This situation is due to the fact that when using wordembedding models, information is lost when converting the vector representations of the words that make up the text into a vector representation of the entire text, which usually has the same dimension as the vector of a single word.

This paper proposes an alternative way for using pre-trained word-embedding models for text vectorization. The essence of the proposed method consists in combining semantically similar elements of the dictionary of the existing text corpus by clustering their (dictionary elements) embeddings, as a result of which a new dictionary is formed with a size smaller than the original one, each element of which corresponds to one cluster. The original corpus of texts is reformulated in terms of this new dictionary, after which vectorization is performed on the reformulated texts using one of the dictionary approaches (TF-IDF was used in the work). The resulting vector representation of the text can be additionally enriched using the vectors of words of the original dictionary obtained by decreasing the dimension of their embeddings for each cluster.

A series of experiments to determine the optimal parameters of the method is described in the paper, the proposed approach is compared with other methods of text vectorization for the text ranking problem - averaging word embeddings with TFIDF weighting and without weighting, as well as vectorization based on TF-IDF coefficients.

Keywords: word embedding; Fasttext; TF-IDF; averaging; clustering; text similarity; distance; text ranking

\section{INFORMATION ABOUT THE AUTHORS}

\begin{tabular}{r|l}
$\begin{array}{r}\text { Vitaly I. Yuferev } \\
\text { correspondence author }\end{array}$ & $\begin{array}{l}\text { orcid.org/0000-0003-3245-6240. E-mail: YuferevVI@mail.cbr.ru } \\
\text { Chief expert, Master of science. }\end{array}$ \\
Nikolai A. Razin & $\begin{array}{l}\text { orcid.org/0000-0002-7669-776X. E-mail: razinna@cbr.ru } \\
\text { Head of division, PhD. }\end{array}$
\end{tabular}

For citation: V. I. Yuferev and N. A. Razin, "Word-embedding Based Text Vectorization Using Clustering”, Modeling and analysis of information systems, vol. 28, no. 3, pp. 292-311, 2021.

(c) Yuferev V. I., Razin N. A., 2021

This is an open access article under the CC BY license (https://creativecommons.org/licenses/by/4.0/). 


\title{
Векторизация текстов на основе word-embedding моделей с использованием кластеризации
}

\author{
В. И. Юферев ${ }^{1}$, Н. А. Разин ${ }^{2}$
}

DOI: $10.18255 / 1818-1015-2021-3-292-311$

${ }^{1}$ Департамент информационных технологий Центрального банка Российской Федерации, Инновационная лаборатория «Новосибирск», ул. Неглинная, д. 12, г. Москва, 107016 Россия.

${ }^{2}$ Департамент противодействия недобросовестным практикам, Центральный банк Российской Федерации, ул. Неглинная, д. 12, г. Москва, 107016 Россия.

УДК 004.8

Научная статья

Полный текст на русском языке
Получена 23 июня 2021 г.

После доработки 16 августа 2021 г.

Принята к публикации 25 августа 2021 г.

Известно, что в задачах обработки естественного языка представление текстов векторами фиксированной длины с использованием word-embedding моделей оправдано в тех случаях, когда векторизуемые тексты являются короткими. Чем сравниваемые тексты длиннее, тем подход работает хуже. Такая ситуация обусловлена тем, что при использовании word-embedding моделей происходит потеря информации при преобразовании векторных представлений слов, составляющих текст, в векторное представление всего текста, имеющее обычно ту же размерность, что и вектор отдельного слова.

В настоящей работе предлагается альтернативный способ использования предобученных word-embedding моделей для векторизации текстов. Суть предлагаемого способа заключается в объединении семантически близких элементов словаря имеющегося корпуса текстов путем кластеризации их (элементов словаря) эмбеддингов, в результате чего формируется новый словарь размером меньше исходного, каждый элемент которого соответствует одному кластеру. Исходный корпус текстов переформулируется в терминах этого нового словаря, после чего на переформулированных текстах выполняется векторизация одним из словарных подходов (в работе применялся TF-IDF). Полученное векторное представление текста дополнительно может обогащаться с использованием векторов слов исходного словаря, полученных путем уменьшения размерности их эмбеддингов по каждому кластеру. В работе описана серия экспериментов по определению оптимальных параметров предлагаемого подхода; для задачи ранжирования текстов приведено сравнение подхода с другими способами векторизации - усреднением эмбеддингов слов со взвешиванием по TF-IDF и без взвешивания, а также с векторизацией на основе TF-IDF коэффициентов.

Ключевые слова: эмбеддинговые модели; Fasttext; TF-IDF; усреднение; кластеризация; семантическое сходство текстов; определение расстояний; ранжирование текстов

\section{ИНФОРМАЦИЯ ОБ АВТОРАХ}

Виталий Иванович Юферев автор для корреспонденции

Николай Алексеевич Разин orcid.org/0000-0003-3245-6240. E-mail: YuferevVI@mail.cbr.ru

Главный эксперт, магистр техники и технологий.

orcid.org/0000-0002-7669-776X. E-mail: razinna@cbr.ru

Начальник отдела, кандидат физ.-мат. наук.

Для цитирования: V. I. Yuferev and N. A. Razin, "Word-embedding Based Text Vectorization Using Clustering”, Modeling and analysis of information systems, vol. 28, no. 3, pp. 292-311, 2021.

() Юферев В. И., Разин Н. А., 2021

Эта статья открытого доступа под лицензией СС BY license (https://creativecommons.org/licenses/by/4.0/). 


\section{Введение}

Распространенной задачей в области обработки естественного языка является ранжирование текстов, то есть определение, какой из двух текстов $\mathrm{T}_{1}$ или $\mathrm{T}_{2}$ семантически ближе к тексту $\mathrm{T}$.

Базовый подход для определения семантического сходства текстов состоит из двух основных этапов: представление сравниваемых текстов в векторном виде, отражающем семантику текста, и последующее определение расстояний между полученными векторами [1].

Существуют различные способы представления текстов в векторном виде: с использованием словарей, с использованием word-embedding моделей, с использованием языковых моделей, основанных на архитектуре Transformer.

К недостаткам словарных подходов, в частности основанных на TF-IDF [2], можно отнести отсутствие учета семантики слов [3] при определении близости текстов, если составляющие их слова не пересекаются, то они будут определены как далекие друг от друга, независимо от того, содержат ли тексты слова, похожие по смыслу. Применение современных языковых моделей, основанных на архитектуре Transformer, также имеет некоторые ограничения: высокие требования к вычислительным ресурсам и предельный размер входа [4-6].

Применение word-embedding моделей для векторизации текстов выглядит оправданным в случаях, когда имеют место указанные выше ограничения других подходов. Особенно актуально применение предобученных word-embedding моделей, когда имеющийся корпус текстов страдает от недостатка данных [7].

Наиболее простой и часто применяемый подход по формированию векторного представления текста с использованием word-embedding моделей заключается в формировании эмбеддингов входящих в текст слов с последующим формированием вектора текста путем усреднения (сложения) полученных эмбеддингов слов [8, 9]. В качестве улучшения подхода дополнительно может выполняться взвешивание полученных векторов эмбеддингов слов, например, по TF-IDF [10].

Решение прикладных задач обработки естественного языка подтверждает, что применение подхода с векторизацией текстов при помощи усреднения (сложения) эмбеддингов слов, как правило, даёт приемлемое качество (конкретные метрики и их значения зависят от задачи) на коротких текстах (на предложениях и меньше) [11] и по мере увеличения длины текстов качество снижается до неприемлемого.

Низкое качество при усреднении (сложении) на длинных текстах можно объяснить следующим. Эмбеддинг слова представляет его семантику в виде вектора. Усреднение (сумма) двух эмбеддингов (обозначим А и В) также представляет собой вектор (обозначим С) той же размерности. При этом возникает неопределенность, связанная с тем, что вектор С может быть получен указанной комбинацией (усреднение, сумма) как исходных векторов А и В, так и некоторых других векторов $\mathrm{D}$ и $\mathrm{E}$, отражающих семантику, отличную от семантики, кодируемой векторами А и В. Соответственно, чем больше исходных эмбеддингов усредняется, тем выше у результирующего вектора неопределенность относительно семантики исходных слов.

Попытка увеличения размерности вектора текста по сравнению с векторами слов приводится в [12-14]. Как и в описываемом в настоящей работе подходе в этих работах предлагается выполнить кластеризацию на словаре корпуса текстов. Однако указанные подходы имеют следующие ограничения: необходимость наличия достаточно большого корпуса текстов для обучения wod2vec модели, отсутствие описания возможности применения для n-грамм.

\section{1. Описание подхода}

Чтобы обойти ограничения алгоритмов по векторизации текстов - словарного и основанного на word-embedding - предлагается совместить данные подходы. 
Попытки совмещения word-embedding и TF-IDF предпринимались и ранее. Суть таких улучшений состоит в использовании при усреднении эмбеддингов слов весовых коэффициентов, соответствующих TF-IDF этих слов. Однако описанная выше во Введении неоднозначность не устраняется, поскольку размерность итогового вектора остается неизменной.

Далее приводится алгоритм формирования векторного представления текста в рамках предлагаемого подхода. При этом необходимо учитывать, что векторизация текста обычно выполняется в рамках решения какой-либо прикладной задачи. Приведенный алгоритм актуален для решения задачи ранжирования текстов (описана во Введении).

1. Имеется исходное множество текстов Т, на которых требуется выполнять ранжирование, то есть для заданного текста $\mathrm{t}_{\mathrm{i}}$ из $\mathrm{T}$ упорядочить множество Т по степени близости к $\mathrm{t}_{\mathrm{i}}$. Также имеется предобученная word-embedding модель $\mathrm{M}$.

2. На множестве T строится словарь $\mathrm{V}$ всех слов, входящих в тексты $\mathrm{t}_{\mathrm{i}}$ из $\mathrm{T}$.

3. Для каждого слова $\mathrm{v}_{\mathrm{i}}$ из словаря $\mathrm{V}$ получаем его эмбеддинг при помощи предобученной модели $\mathrm{M}: \mathrm{e}_{\mathrm{i}}=\mathrm{M}\left(\mathrm{v}_{\mathrm{i}}\right)$. Все $\mathrm{e}_{\mathrm{i}}$ в совокупности составляют множество эмбеддингов Е.

4. Выполняется кластеризация на множестве Е, в результате которой получается кластеризующая модель С, которая по эмбеддингу слова выдает кластер, к которому он относится. Для каждого $\mathrm{e}_{\mathrm{i}}$ из $\mathrm{E}$ определяется его кластер $\mathrm{c}_{\mathrm{i}}=\mathrm{C}\left(\mathrm{e}_{\mathrm{i}}\right)$. Множество всех кластеров $\mathrm{c}_{\mathrm{i}}$ модели С также обозначим символом С.

5. Для каждого текста $t_{i}$ из $\mathrm{T}$ получаем новый текст $\mathrm{t}^{\mathrm{c}}{ }_{\mathrm{i}}$ следующим способом:

5.1. Копируем $t_{i}$ в $t^{c}{ }_{i}$;

5.2. Для каждого слова $\mathrm{w}_{\mathrm{j}}$ из текста $\mathrm{t}^{\mathrm{c}}{ }_{\mathrm{i}}$

a) определяем его кластер $\mathrm{c}_{\mathrm{j}}=\mathrm{C}\left(\mathrm{M}\left(\mathrm{w}_{\mathrm{j}}\right)\right)$;

b) заменяем в тексте $\mathrm{t}_{\mathrm{i}}^{\mathrm{c}}$ слово $\mathrm{w}_{\mathrm{j}}$ на номер соответствующего кластера $\mathrm{c}_{\mathrm{j}}$.

В результате путем замены всех $\mathrm{t}_{\mathrm{i}}$ из $\mathrm{T}$ на $\mathrm{t}^{\mathrm{c}}{ }_{\mathrm{i}}$ получено новое множество $\mathrm{T}_{\mathrm{c}}$. Bсе тексты этого множества состоят из номеров кластеров с символами-разделителями между ними.

6. Выполняется векторизация текстов $\mathrm{T}_{\mathrm{c}}$ при помощи TF-IDF на $\mathrm{n}$-граммах слов. В результате чего получается:

- множество $\mathrm{X}_{\mathrm{c}}$ TF-IDF-векторов $\mathrm{x}_{\mathrm{i}}^{\mathrm{c}}$ для каждого $\mathrm{t}_{\mathrm{i}}^{\mathrm{c}}$ из $\mathrm{T}_{\mathrm{c}}$,

- словарь n-грамм $\mathrm{V}_{\mathrm{c}}$, а также

- $\mathrm{N}_{\min }$ и $\mathrm{N}_{\max }$ - заданные в качестве входных параметров алгоритма минимальная и максимальная длины n-грамм, используемых для построения словаря TF-IDF.

7. Для каждого $t_{i}$ из $\mathrm{T}^{\mathrm{c}}{ }_{\mathrm{i}}$ из $\mathrm{X}_{\mathrm{c}}$ далее рассматривается как векторное представление текста $\mathrm{t}_{\mathrm{i}}$.

Поскольку объединенные в один кластер одни слова исходного словаря могут быть ближе друг к другу, чем другие, обогащение векторного представления информацией о взаимной близости слов кластера может повысить качество этого векторного представления. Чтобы учесть в векторном представлении текстов взаимную близость слов друг к другу в рамках одного кластера, предлагается следующее улучшение подхода в виде дополнительных шагов алгоритма.

Формирование обогащающих векторов слов.

8. По каждому кластеру $\mathrm{c}_{\mathrm{i}}$ из С.

8.1. Для каждого относящегося к $c_{i}$ эмбеддинга $\mathrm{e}_{\mathrm{j}}$ слова $\mathrm{w}_{\mathrm{j}}$ исходного словаря V выполняется снижение размерности до одного (располагаются на одной числовой оси). Полученные в результате числовые значения обозначим через $\mathrm{e}^{\mathrm{r}}{ }_{\mathrm{j}}$.

8.2. С использованием min-max-нормализации выполняется масштабирование числовых представлений $\mathrm{e}_{\mathrm{j}}^{\mathrm{r}}$ эмбеддингов $\mathrm{e}_{\mathrm{j}}$ слов $\mathrm{w}_{\mathrm{j}}$ кластера $\mathrm{c}_{\mathrm{i}}$ на отрезок [0;1]. Отрезок [0;1] разбивается на D-1 частей (D - размерность обогащающего вектора слова, задается в качестве одного из входных параметров алгоритма), пронумерованных от 1 до D-1. 
8.3. Для каждого слова $\mathrm{w}_{\mathrm{j}}$ исходного словаря $\mathrm{V}$ выполняется формирование обогащающего вектора $\mathrm{e}_{\mathrm{j}}^{\mathrm{e}}$ следующим образом. Нулевая позиция вектора всегда заполняется значением «1». Далее по каждому из D-1 отрезков, на которые разбит интервал [0;1], если е ${ }_{\mathrm{j}}^{\mathrm{j}}$ попадает в k-й интервал, то k-я позиция вектора заполняется значением « $1 »$, иначе «0».

Формирование обогащенных векторов текстов.

9. Для каждого текста $\mathrm{t}_{\mathrm{i}}^{\mathrm{c}}$ из $\mathrm{T}_{\mathrm{c}}$.

Каждая позиция (обозначим индексом j) TF-IDF вектора $\mathrm{x}_{\mathrm{i}}^{\mathrm{c}}$

соответствует TF-IDF-коэффициенту $\mathrm{x}_{\mathrm{ij}}^{\mathrm{c}}$ для n-граммы $\mathrm{w}^{\mathrm{c}}$ из словаря $\mathrm{V}_{\mathrm{c}}$. Для каждой j-й позиции вектора $\mathrm{x}_{\mathrm{i}}^{\mathrm{c}}$ сформируем обогащающий вектор $\mathrm{e}^{\mathrm{xc}}{ }_{\mathrm{j}}$ следующим образом.

9.1. На основе текста $t_{i}$ из $\mathrm{T}$ сформируем обогащающие векторы всех входящих в $t_{i} n$-грамм длиной от $\mathrm{N}_{\min }$ до $\mathrm{N}_{\max }$ путем конкатенации обогащающих векторов входящих в них слов (полученных на шаге 8.3). Максимальная длина каждого такого вектора равна $\mathrm{N}_{\max }{ }^{*} \mathrm{D}$. Если вектор получен из n-граммы длиной меньше $\mathrm{N}_{\max }$, вектор дополняется справа нулями до максимальной длины.

9.2. Каждой $\mathrm{n}$-грамме из $\mathrm{t}_{\mathrm{i}}$ соответствует некоторая $\mathrm{n}$-грамма из $\mathrm{V}_{\mathrm{c}}$. Выполним усреднение обогащающих векторов $\mathrm{n}$-грамм из $\mathrm{t}_{\mathrm{i}}$ по соответствующим им $\mathrm{n}$-граммам из $\mathrm{V}_{\mathrm{c}}$. Таким образом, получены обогащающие векторы для тех позиций вектора $\mathrm{x}^{\mathrm{c}}$, для которых соответствующие n-граммы $\mathrm{w}^{\mathrm{c}}{ }_{\mathrm{j}}$ из $\mathrm{V}_{\mathrm{c}}$ входят в $\mathrm{t}^{\mathrm{c}}{ }_{\mathrm{i}}$.

9.3. Если соответствующие $\mathrm{j}$-й позиции из $\mathrm{x}_{\mathrm{c}}^{\mathrm{c}} \mathrm{n}$-граммы отсутствуют в $\mathrm{t}_{\mathrm{j}}^{\mathrm{c}}$, то соответствующие обогащающие векторы состоят из $\mathrm{N}_{\max }{ }^{*} \mathrm{D}$ нулевых элементов.

9.4. Обогащенный вектор $\mathrm{x}^{\mathrm{ce}}{ }_{\mathrm{i}}$ текста $\mathrm{t}_{\mathrm{i}}$ вычисляется конкатенацией векторов $\mathrm{x}_{\mathrm{ij}}{ }^{*} \mathrm{e}^{\mathrm{xc}}{ }_{\mathrm{j}}$ (произведение TF-IDF-коэффициента n-граммы w ${ }^{c}$ на ее обогащающий вектор).

Таким образом, получено множество обогащенных векторных представлений $\mathrm{X}_{\text {се }}$ текстов $\mathrm{T}$.

\section{2. Апробация подхода}

\section{1. Условия проведения апробации}

Проверка качества подхода по векторизации текстов осуществлялась в контексте решения задачи ранжирования текстов по семантической близости.

Для проверки качества подхода имелось в распоряжении 13772 примера вида: $\left(\mathrm{T}_{1}, \mathrm{~T}_{2}, \mathrm{~T}\right)$, где $\mathrm{T}_{1}$, $\mathrm{T}_{2}$ и $\mathrm{T}-$ тексты, такие что $\mathrm{T}_{1}$ ближе к $\mathrm{T}$, чем $\mathrm{T}_{2}$.

Все тексты из 13772 примеров сформированы на основе 940 текстов, представляющих собой внутреннюю переписку в Банке России.

Качество подхода по ранжированию текстов определяется по точности (Accuracy) как отношение количества корректно определенных примеров к общему их количеству.

Распределение длин текстов корпуса представлено на рисунке 1. По горизонтальной оси отложены длины текстов в символах. По вертикальной - количество текстов заданной длины.

Тексты корпуса предварительно были приведены к нижнему регистру, из них были отфильтрованы символы, не являющиеся пробелом или буквами русского или латинского алфавитов.

Размер словаря корпуса текстов составляет 73731 слово.

В качестве меры, при помощи которой определяется близость между векторными представлениями текстов, использовалась косинусная близость.

\section{2. Определение оптимальных параметров}

\subsection{1. Параметры алгоритма}

В рамках предлагаемого подхода: 


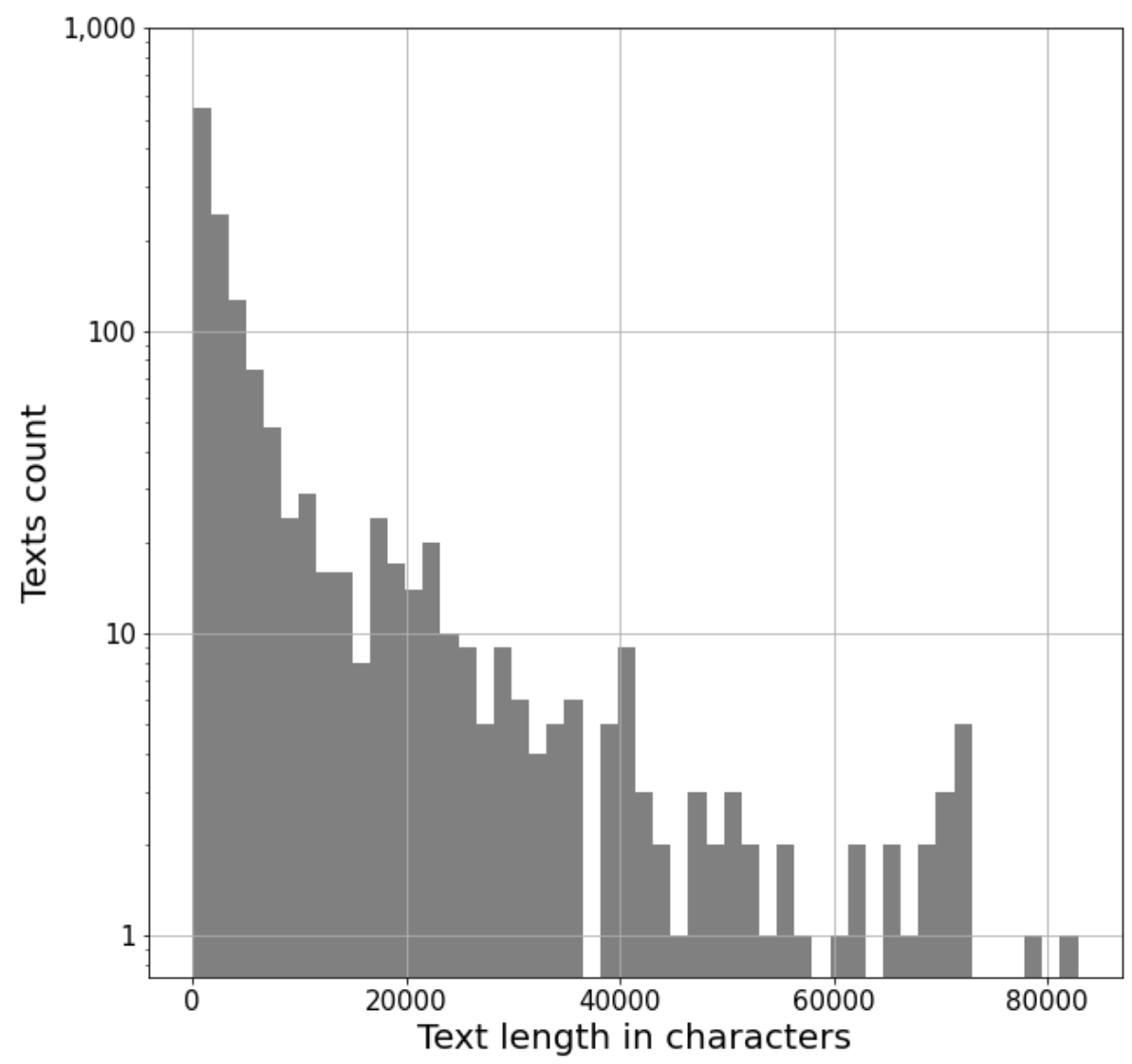

Fig. 1. Distribution of text lengths in the corpus

Рис. 1. Распределение длин текстов в корпусе

- в качестве word-embedding модели использовалась FastText модель от DeepPavlov' ${ }^{1}$, обученная на русскоязычной части Wikipedia совместно с набором данных Lenta ru news. Размерность выходного вектора 300. Использованная в работе модель Fasttext формирует эмбеддинг слова с использованием входящих в это слово символьных последовательностей, что позволяет применять ее к словам out-of-vocabulary, то есть таким, которые в обучении FastText модели не участвовали.

- в качестве метода кластеризации использован Kmeans,

- в качестве метода снижения размерности на шаге 8.1 использован t-SNE,

- при векторизации TF-IDF устанавливается фильтр на минимальное количество документов, в которых встречается n-грамма, равная двум.

Изменяемыми параметрами для алгоритма являются: количество кластеров, диапазон n-грамм, размерность обогащающего вектора слова.

Применим предлагаемый подход для всех возможных комбинаций параметров из представленных в Таблице 1, выполнив серию экспериментов по три для каждой комбинации параметров. Здесь размерность обогащающего вектора слова, равная нулю, означает, что обогащение не производится, а используются непосредственно TF-IDF-векторы.

\footnotetext{
${ }^{1} \mathrm{http} / / /$ files.deeppavlov.ai/embeddings/ft_native_300_ru_wiki_lenta_lower_case/ft_native_300_ru_wiki_lenta_lower_case.bin
} 
Table 1. The values of the parameters to be checked
Таблица 1. Проверяемые значения параметров алгоритма

\begin{tabular}{|l|l|}
\hline Параметр & Значения \\
\hline Количество кластеров & $\begin{array}{l}100,1000,3000,5000,10000,15000,20000,25000, \\
45000,65000\end{array}$ \\
\hline Размерность обогащающего вектора слова & $0,2,5,10,15,20$ \\
\hline Диапазон n-грамм & $(1,1),(1,2),(1,3),(1,4)$ \\
\hline
\end{tabular}

\subsection{2. Результаты}

Таблица с полными результатами экспериментов приведена в Приложении А.

Значения параметров, на которых получены лучшие показатели точности для различных диапазонов n-грамм, представлены в Таблице 2.

Из таблицы видно, что лучшие результаты получены для следующей комбинации параметров: количество кластеров 25000, размер обогащающего вектора слова 2, диапазон n-грамм $(1,4)$.

Table 2. Parameters that give the best results

Таблица 2. Параметры с лучшими результатами

\begin{tabular}{|r|r|r|r|r|}
\hline $\begin{array}{r}\text { диапазон } \\
\text { n-gram }\end{array}$ & $\begin{array}{r}\text { Количество } \\
\text { кластеров }\end{array}$ & $\begin{array}{r}\text { Размерность } \\
\text { обогащающего } \\
\text { вектора слова }\end{array}$ & Точность & $\begin{array}{r}\text { Стандартное } \\
\text { отклонение }\end{array}$ \\
\hline$(1,1)$ & 20000 & 0 & 0.934 & 1.321 \\
\hline$(1,2)$ & 25000 & 0 & 0,940 & 1.151 \\
\hline$(1,3)$ & 25000 & 2 & 0.944 & 1.156 \\
\hline$(1,4)$ & 25000 & 2 & $\mathbf{0 . 9 4 7}$ & 1.16 \\
\hline
\end{tabular}

\subsection{3. Интерпретация результатов}

Отметим, что значение в 25000 кластеров, на котором получено лучшее значение точности, составляет приблизительно одну третью часть от размера словаря корпуса текстов (73731 слово).

Ниже приведены графики зависимости точности от параметров алгоритма.

На Рисунке 2 приведен график зависимости точности от количества кластеров для разной размерности обогащающего вектора слова. Значения точности для графика получены усреднением точности для всех диапазонов n-грамм.

Из графика видно, что увеличение количества кластеров до определенного значения приводит к значительному повышению точности. При количестве кластеров 25000 точность достигает максимального значения, после чего начинает убывать.

Завершается график горизонтальным участком, что можно объяснить следующим: чем ближе параметр «количество кластеров» при кластеризации к количеству кластеризуемых объектов, тем больше получается «пустых» кластеров, то есть, несмотря на увеличение значения параметра «количество кластеров», словарь $\mathrm{V}_{\mathrm{c}}$ описанного алгоритма растет незначительно.

Зависимость точности от размерности обогащающего вектора слова имеет разный характер, в зависимости от количества кластеров, в связи с чем для зависимости точности от размерности обогащающего вектора слова приводятся два графика: график для количества кластеров до 20000 приведен на Рисунке 3, а график для количества кластеров 20000 и более приведен на Рисунке 4. Значения точности для графиков получены усреднением точности для всех диапазонов n-грамм.

Из графиков видно, что чем меньше количество кластеров, тем больший прирост точности дает процедура обогащения, а начиная с определенного количества кластеров, в целом, обогащение понижает точность. При этом наиболее существенный прирост точности наблюдается на размерности, равной двум. Также видно, что при использовании обогащения максимальный прирост точности на малом количестве кластеров больше, чем максимальное снижение на большом. 


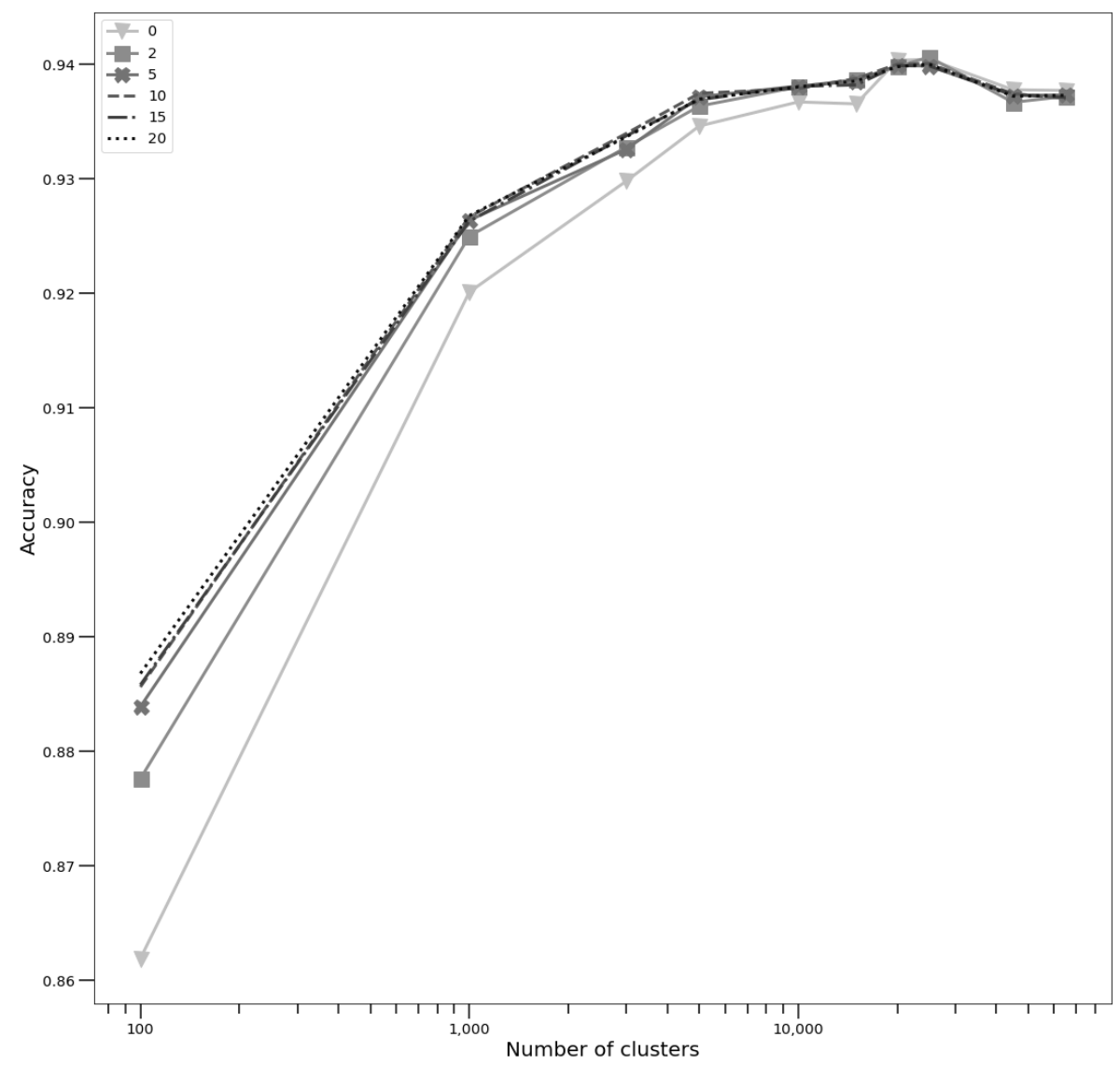

Fig. 2. Dependence of accuracy on the number of clusters
Рис. 2. Зависимость точности от количества кластеров

График зависимости точности от количества кластеров для различных диапазонов используемых n-грамм приведен на Рисунке 5. Значения точности для графика получены усреднением точности для всех размерностей обогащающего вектора. Из графика видно, что чем больше используемый диапазон n-грамм, тем точность выше.

\section{3. Сравнение с baseline-подходами}

Сравниваются следующие подходы:

- предлагаемый в настоящей работе (обогащенные TF-IDF-векторы на номерах кластеров),

- TF-IDF на текстах корпуса,

- TF-IDF на лемматизированных текстах корпуса,

- усреднение эмбеддингов,

- усреднение эмбеддингов, взвешенных по TF-IDF. 


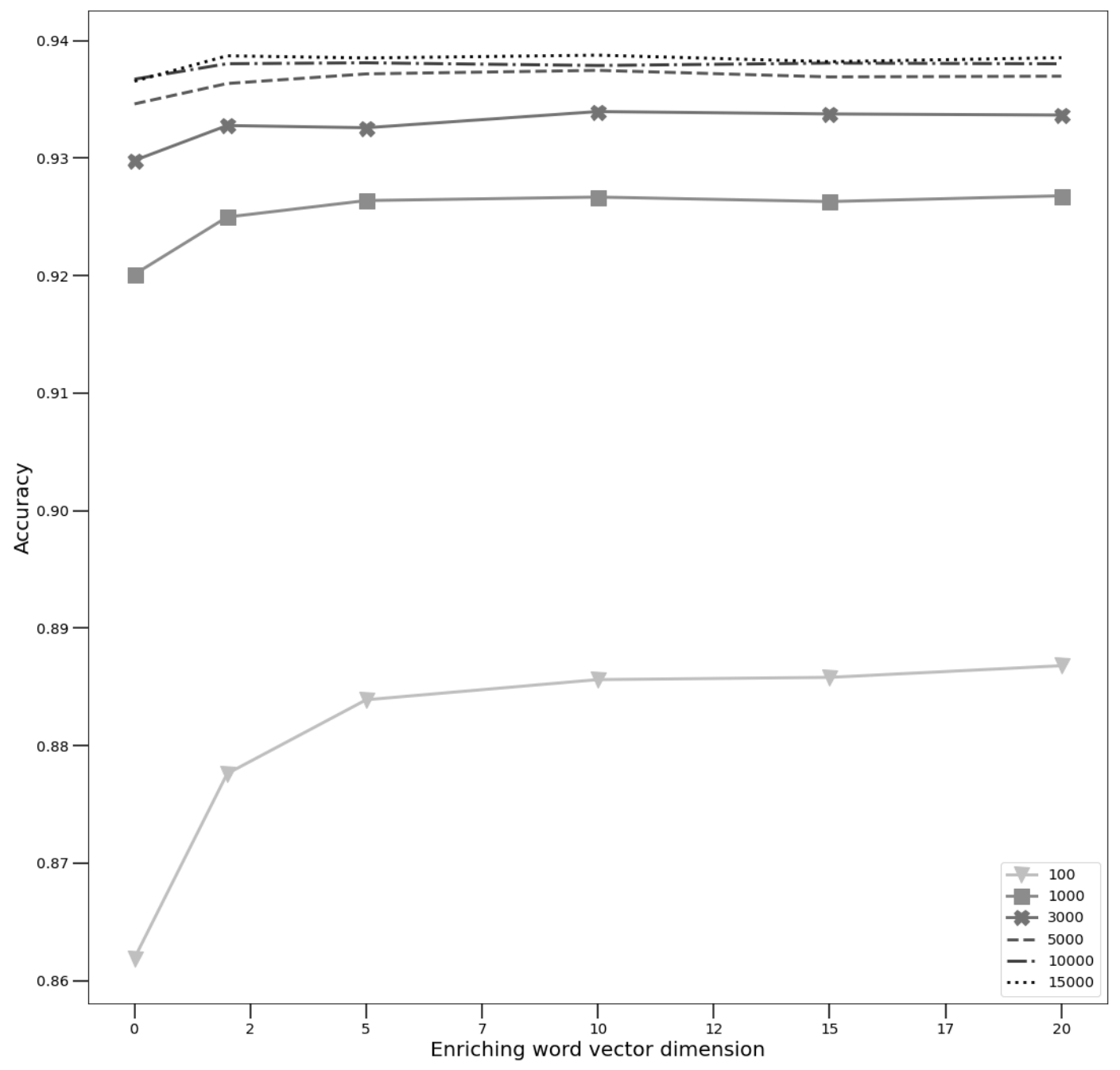

Fig. 3. Dependence of accuracy on enriching word vector for the number of clusters less than 20,000
Рис. 3. Зависимость точности от размерности обогащающего вектора слова для количества кластеров менее 20000

\subsubsection{TF-IDF}

Векторизация текстов на основе TF-IDF для следующих диапазонов n-грамм: $(1,1),(1,2),(1,3)$, $(1,4)$.

Векторизация TF-IDF осуществлялась с использованием библиотеки sklearn.

При векторизации TF-IDF установлен фильтр на минимальное количество документов, в которых встречается n-грамма, равная двум. 


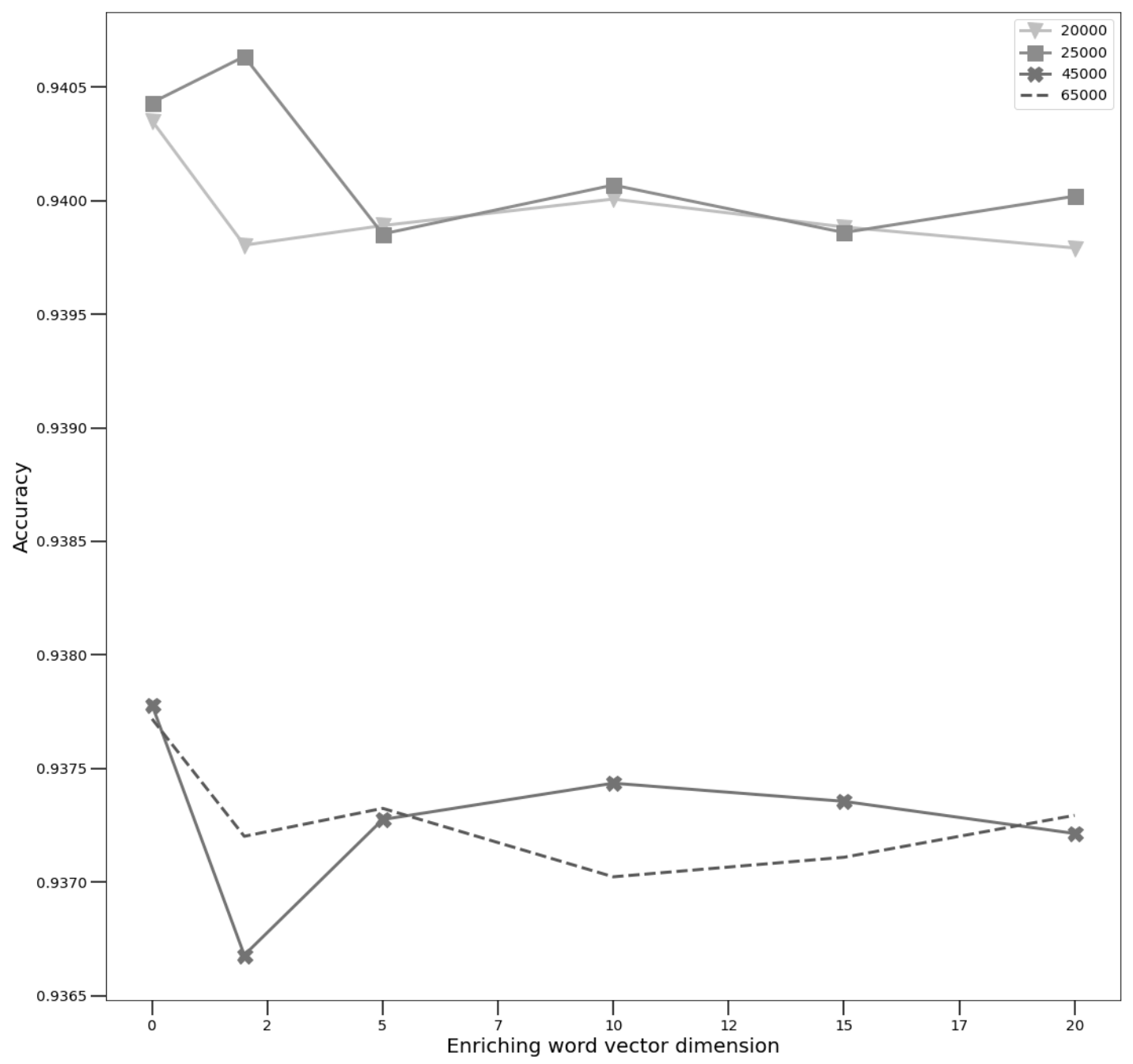

Fig. 4. Dependence of accuracy on the dimension of the enriching word vector for the number of clusters of 20,000 or more
Рис. 4. Зависимость точности от размерности обогащающего вектора слова для количества кластеров 20000 и более

\subsubsection{TF-IDF с лемматизацией}

Практика решения задач обработки естественного языка показывает, что поскольку в русском языке разные формы слова часто имеют разное написание, непосредственное применение подхода TF-IDF для векторизации текстов в большинстве случаев работает хуже, чем в случае, если выполнено предварительное приведение слов текста в нормальную форму.

В эксперименте приведение слов в нормальную форму выполнялось путем лемматизации при помощи библиотеки Mystem от компании Яндекс. 


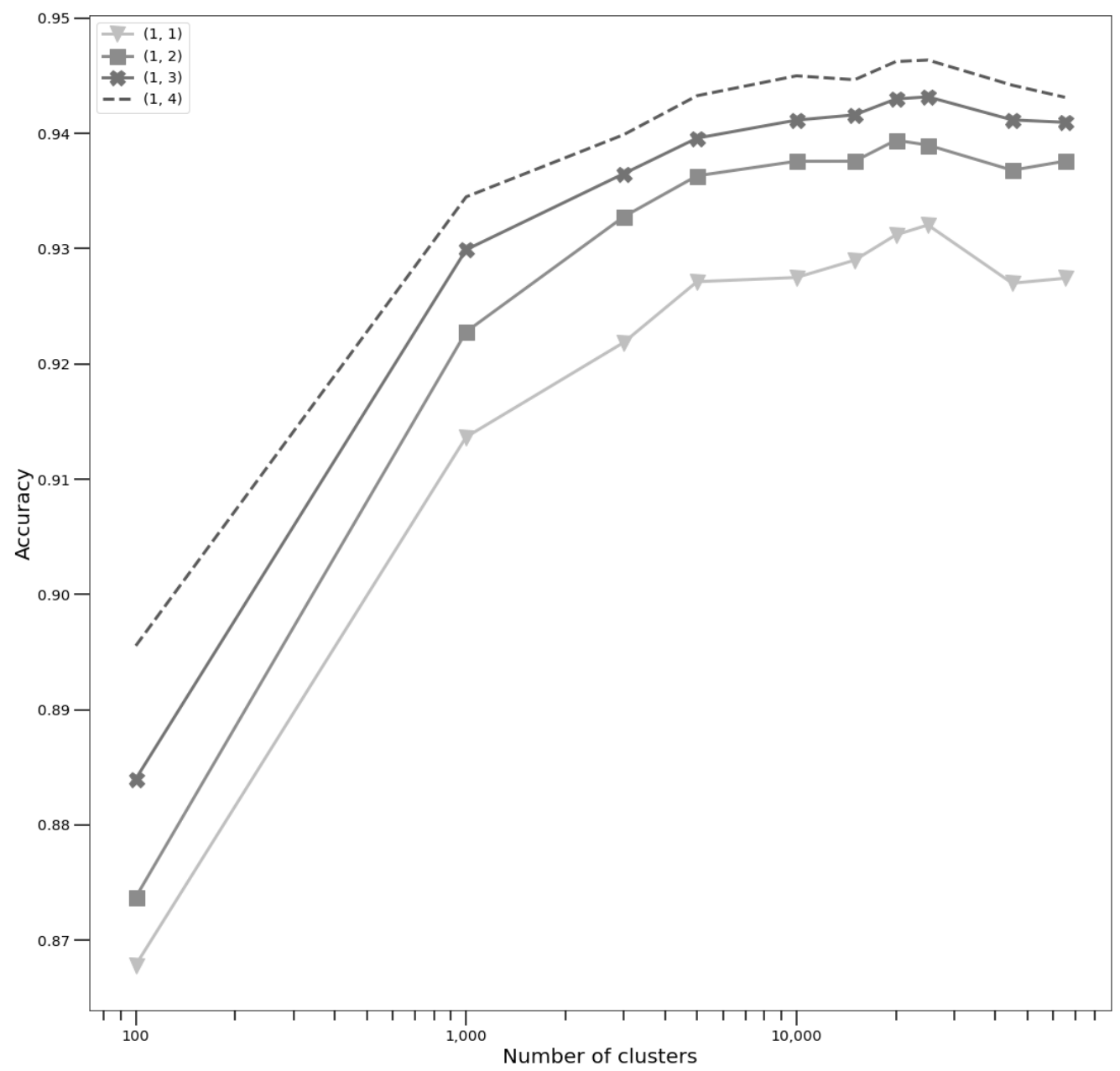

Fig. 5. Dependence of accuracy on the number of clusters for different n-gram ranges
Рис. 5. Зависимость точности от количества кластеров для разных диапазонов n-грамм

Векторизация текстов на основе TF-IDF выполнялась для следующих видов n-грамм: $(1,1),(1,2)$, $(1,3),(1,4)$.

Векторизация TF-IDF осуществлялась с использованием библиотеки sklearn.

При векторизации TF-IDF установлен фильтр на минимальное количество документов, в которых встречается n-грамма, равная двум.

\subsection{3. Усреднение эмбеддингов}

Усреднение эмбеддингов для текста заключается в преобразовании каждого слова текста в векторное представление при помощи word-embedding модели и последующем усреднении эмбеддингов слов с получением итогового вектора текста той же размерности, что и векторы эмбеддингов. 


\subsection{4. Усреднение эмбеддингов, взвешенных по TF-IDF}

Подход отличается от простого усреднения эмбеддингов тем, что при усреднении эмбеддинги слов берутся с весовыми коэффициентами, соответствующими их TF-IDF-коэффициентам, рассчитанным на имеющемся корпусе текстов [10]. В настоящей работе коэффициенты TF-IDF рассчитывались на всем корпусе документов.

\subsection{5. Результаты сравнения подходов}

Результаты сравнения подходов приведены в таблице 3.

Table 3. Methods comparison

Таблица 3. Сравнение подходов

\begin{tabular}{|l|r|r|l|l|r|}
\hline $\begin{array}{l}\text { Диапазон } \\
\text { n-грамм }\end{array}$ & TF-IDF & $\begin{array}{l}\text { TF-IDF со } \\
\text { стеммингом }\end{array}$ & $\begin{array}{l}\text { Усреднение } \\
\text { эмбеддингов } \\
\text { Fasttext }\end{array}$ & $\begin{array}{l}\text { Усреднение } \\
\text { эмбеддингов } \\
\text { Fasttext coxt } \\
\text { взвешиванием } \\
\text { по TD-IDF }\end{array}$ & $\begin{array}{l}\text { Обогащенные TF- } \\
\text { IDF векторы на } \\
\text { кластерах }\end{array}$ \\
\hline$(1,1)$ & 0,9287 & 0,9279 & 0,779 & 0,8679 & 0.9302 \\
\hline$(1,2)$ & 0,9333 & 0,9364 & не применимо & не применимо & 0.9405 \\
\hline$(1,3)$ & 0,9366 & 0,9396 & не применимо & не применимо & 0.9459 \\
\hline$(1,4)$ & 0,9381 & 0,94 & не применимо & не применимо & 0.947 \\
\hline
\end{tabular}

\section{Заключение}

В настоящей работе предложен подход по векторизации текстов при помощи word-embedding моделей, экспериментально определены оптимальные параметры для решения задачи ранжирования текстов, представляющих собой 940 писем внутренней переписки Банка России, выполнено сравнение предложенного подхода с распространенными подходами.

Предлагаемый подход в сравнении с представленными baseline-подходами показывает лучшие результаты.

Использовавшийся в предлагаемом подходе алгоритм Kmeans для кластеризации эмбеддингов элементов словаря в качестве входного параметра принимает количество кластеров. При этом остается открытым вопрос, насколько компактными получаются кластеры, что может влиять на качество представления текста при помощи кластеров.

Для решения данной проблемы видятся перспективными следующие направления дальнейших исследований:

- Анализ компактности получаемых кластеров для улучшения представления текстов в виде номеров кластеров, например, фильтрация элементов кластера по порогу расстояния до центроида.

- Использование такого подхода для кластеризации, при котором параметры определяют расстояния между объектами, а не количество кластеров. Однако, по сравнению с Kmeans, такие подходы более требовательны к вычислительным ресурсам. Соответственно, в контексте данного направления актуально решение задачи поиска эффективного способа кластеризации элементов словаря.

Предложенный подход для векторизации апробирован в рамках решения задачи ранжирования текстов. Необходимо исследовать подход на применимость для решения других задач обработки естественного языка. 


\section{Appendix A. Experimental results}

\section{Приложение А. Результаты экспериментов}

\begin{tabular}{|c|c|c|c|c|c|}
\hline $\begin{array}{r}\text { № } \\
\mathbf{\Pi} / \mathbf{\Pi}\end{array}$ & $\begin{array}{r}\text { Количество } \\
\text { кластеров }\end{array}$ & $\begin{array}{r}\text { Диапазон } \\
\text { n-грамм }\end{array}$ & $\begin{array}{r}\text { Размерность } \\
\text { вектора } \\
\text { обогащения } \\
\text { слова }\end{array}$ & Точность & $\begin{array}{r}\text { Стандартное } \\
\text { отклонение }\end{array}$ \\
\hline 1 & 100 & $(1,1)$ & 0 & 0,858 & 1,051 \\
\hline 2 & 100 & $(1,1)$ & 2 & 0,863 & 1,058 \\
\hline 3 & 100 & $(1,1)$ & 5 & 0,87 & 1,066 \\
\hline 4 & 100 & $(1,1)$ & 10 & 0,871 & 1,067 \\
\hline 5 & 100 & $(1,1)$ & 15 & 0,871 & 1,067 \\
\hline 6 & 100 & $(1,1)$ & 20 & 0,873 & 1,069 \\
\hline 7 & 100 & $(1,2)$ & 0 & 0,86 & 1,054 \\
\hline 8 & 100 & $(1,2)$ & 2 & 0,871 & 1,066 \\
\hline 9 & 100 & $(1,2)$ & 5 & 0,877 & 1,074 \\
\hline 10 & 100 & $(1,2)$ & 10 & 0,878 & 1,075 \\
\hline 11 & 100 & $(1,2)$ & 15 & 0,878 & 1,075 \\
\hline 12 & 100 & $(1,2)$ & 20 & 0,879 & 1,076 \\
\hline 13 & 100 & $(1,3)$ & 0 & 0,862 & 1,056 \\
\hline 14 & 100 & $(1,3)$ & 2 & 0,882 & 1,08 \\
\hline 15 & 100 & $(1,3)$ & 5 & 0,888 & 1,087 \\
\hline 16 & 100 & $(1,3)$ & 10 & 0,89 & 1,09 \\
\hline 17 & 100 & $(1,3)$ & 15 & 0,891 & 1,091 \\
\hline 18 & 100 & $(1,3)$ & 20 & 0,892 & 1,092 \\
\hline 19 & 100 & $(1,4)$ & 0 & 0,867 & 1,062 \\
\hline 20 & 100 & $(1,4)$ & 2 & 0,895 & 1,096 \\
\hline 21 & 100 & $(1,4)$ & 5 & 0,901 & 1,104 \\
\hline 22 & 100 & $(1,4)$ & 10 & 0,903 & 1,106 \\
\hline 23 & 100 & $(1,4)$ & 15 & 0,903 & 1,106 \\
\hline 24 & 100 & $(1,4)$ & 20 & 0,904 & 1,107 \\
\hline 25 & 1000 & $(1,1)$ & 0 & 0,91 & 1,115 \\
\hline 26 & 1000 & $(1,1)$ & 2 & 0,912 & 1,118 \\
\hline 27 & 1000 & $(1,1)$ & 5 & 0,915 & 1,121 \\
\hline 28 & 1000 & $(1,1)$ & 10 & 0,915 & 1,12 \\
\hline 29 & 1000 & $(1,1)$ & 15 & 0,915 & 1,12 \\
\hline 30 & 1000 & $(1,1)$ & 20 & 0,915 & 1,12 \\
\hline 31 & 1000 & $(1,2)$ & 0 & 0,918 & 1,125 \\
\hline 32 & 1000 & $(1,2)$ & 2 & 0,923 & 1,13 \\
\hline 33 & 1000 & $(1,2)$ & 5 & 0,924 & 1,131 \\
\hline 34 & 1000 & $(1,2)$ & 10 & 0,924 & 1,132 \\
\hline 35 & 1000 & $(1,2)$ & 15 & 0,924 & 1,131 \\
\hline 36 & 1000 & $(1,2)$ & 20 & 0,924 & 1,132 \\
\hline 37 & 1000 & $(1,3)$ & 0 & 0,924 & 1,132 \\
\hline 38 & 1000 & $(1,3)$ & 2 & 0,93 & 1,139 \\
\hline 39 & 1000 & $(1,3)$ & 5 & 0,931 & 1,14 \\
\hline
\end{tabular}




\begin{tabular}{|c|c|c|c|c|c|}
\hline 40 & 1000 & $(1,3)$ & 10 & 0,932 & 1,141 \\
\hline 41 & 1000 & $(1,3)$ & 15 & 0,931 & 1,14 \\
\hline 42 & 1000 & $(1,3)$ & 20 & 0,931 & 1,141 \\
\hline 43 & 1000 & $(1,4)$ & 0 & 0,928 & 1,136 \\
\hline 44 & 1000 & $(1,4)$ & 2 & 0,935 & 1,145 \\
\hline 45 & 1000 & $(1,4)$ & 5 & 0,936 & 1,146 \\
\hline 46 & 1000 & $(1,4)$ & 10 & 0,936 & 1,147 \\
\hline 47 & 1000 & $(1,4)$ & 15 & 0,936 & 1,146 \\
\hline 48 & 1000 & $(1,4)$ & 20 & 0,937 & 1,147 \\
\hline 49 & 3000 & $(1,1)$ & 0 & 0,92 & 1,126 \\
\hline 50 & 3000 & $(1,1)$ & 2 & 0,921 & 1,128 \\
\hline 51 & 3000 & $(1,1)$ & 5 & 0,922 & 1,129 \\
\hline 52 & 3000 & $(1,1)$ & 10 & 0,923 & 1,131 \\
\hline 53 & 3000 & $(1,1)$ & 15 & 0,923 & 1,13 \\
\hline 54 & 3000 & $(1,1)$ & 20 & 0,923 & 1,13 \\
\hline 55 & 3000 & $(1,2)$ & 0 & 0,929 & 1,138 \\
\hline 56 & 3000 & $(1,2)$ & 2 & 0,933 & 1,142 \\
\hline 57 & 3000 & $(1,2)$ & 5 & 0,932 & 1,142 \\
\hline 58 & 3000 & $(1,2)$ & 10 & 0,934 & 1,144 \\
\hline 59 & 3000 & $(1,2)$ & 15 & 0,934 & 1,144 \\
\hline 60 & 3000 & $(1,2)$ & 20 & 0,934 & 1,144 \\
\hline 61 & 3000 & $(1,3)$ & 0 & 0,934 & 1,143 \\
\hline 62 & 3000 & $(1,3)$ & 2 & 0,937 & 1,148 \\
\hline 63 & 3000 & $(1,3)$ & 5 & 0,936 & 1,147 \\
\hline 64 & 3000 & $(1,3)$ & 10 & 0,937 & 1,148 \\
\hline 65 & 3000 & $(1,3)$ & 15 & 0,938 & 1,148 \\
\hline 66 & 3000 & $(1,3)$ & 20 & 0,937 & 1,148 \\
\hline 67 & 3000 & $(1,4)$ & 0 & 0,937 & 1,147 \\
\hline 68 & 3000 & $(1,4)$ & 2 & 0,94 & 1,151 \\
\hline 69 & 3000 & $(1,4)$ & 5 & 0,94 & 1,151 \\
\hline 70 & 3000 & $(1,4)$ & 10 & 0,941 & 1,152 \\
\hline 71 & 3000 & $(1,4)$ & 15 & 0,941 & 1,152 \\
\hline 72 & 3000 & $(1,4)$ & 20 & 0,941 & 1,152 \\
\hline 73 & 5000 & $(1,1)$ & 0 & 0,926 & 1,134 \\
\hline 74 & 5000 & $(1,1)$ & 2 & 0,927 & 1,135 \\
\hline 75 & 5000 & $(1,1)$ & 5 & 0,927 & 1,136 \\
\hline 76 & 5000 & $(1,1)$ & 10 & 0,928 & 1,137 \\
\hline 77 & 5000 & $(1,1)$ & 15 & 0,927 & 1,135 \\
\hline 78 & 5000 & $(1,1)$ & 20 & 0,927 & 1,136 \\
\hline 79 & 5000 & $(1,2)$ & 0 & 0,934 & 1,144 \\
\hline 80 & 5000 & $(1,2)$ & 2 & 0,936 & 1,147 \\
\hline 81 & 5000 & $(1,2)$ & 5 & 0,937 & 1,148 \\
\hline 82 & 5000 & $(1,2)$ & 10 & 0,937 & 1,148 \\
\hline 83 & 5000 & $(1,2)$ & 15 & 0,937 & 1,147 \\
\hline 84 & 5000 & $(1,2)$ & 20 & 0,936 & 1,147 \\
\hline
\end{tabular}




\begin{tabular}{|c|c|c|c|c|c|}
\hline 85 & 5000 & $(1,3)$ & 0 & 0,937 & 1,148 \\
\hline 86 & 5000 & $(1,3)$ & 2 & 0,94 & 1,151 \\
\hline 87 & 5000 & $(1,3)$ & 5 & 0,94 & 1,151 \\
\hline 88 & 5000 & $(1,3)$ & 10 & 0,941 & 1,152 \\
\hline 89 & 5000 & $(1,3)$ & 15 & 0,94 & 1,151 \\
\hline 90 & 5000 & $(1,3)$ & 20 & 0,94 & 1,151 \\
\hline 91 & 5000 & $(1,4)$ & 0 & 0,941 & 1,152 \\
\hline 92 & 5000 & $(1,4)$ & 2 & 0,943 & 1,155 \\
\hline 93 & 5000 & $(1,4)$ & 5 & 0,944 & 1,156 \\
\hline 94 & 5000 & $(1,4)$ & 10 & 0,944 & 1,156 \\
\hline 95 & 5000 & $(1,4)$ & 15 & 0,944 & 1,156 \\
\hline 96 & 5000 & $(1,4)$ & 20 & 0,944 & 1,156 \\
\hline 97 & 10000 & $(1,1)$ & 0 & 0,928 & 1,137 \\
\hline 98 & 10000 & $(1,1)$ & 2 & 0,927 & 1,135 \\
\hline 99 & 10000 & $(1,1)$ & 5 & 0,928 & 1,137 \\
\hline 100 & 10000 & $(1,1)$ & 10 & 0,927 & 1,136 \\
\hline 101 & 10000 & $(1,1)$ & 15 & 0,927 & 1,136 \\
\hline 102 & 10000 & $(1,1)$ & 20 & 0,927 & 1,136 \\
\hline 103 & 10000 & $(1,2)$ & 0 & 0,936 & 1,147 \\
\hline 104 & 10000 & $(1,2)$ & 2 & 0,938 & 1,149 \\
\hline 105 & 10000 & $(1,2)$ & 5 & 0,938 & 1,148 \\
\hline 106 & 10000 & $(1,2)$ & 10 & 0,938 & 1,149 \\
\hline 107 & 10000 & $(1,2)$ & 15 & 0,938 & 1,149 \\
\hline 108 & 10000 & $(1,2)$ & 20 & 0,938 & 1,148 \\
\hline 109 & 10000 & $(1,3)$ & 0 & 0,94 & 1,151 \\
\hline 110 & 10000 & $(1,3)$ & 2 & 0,942 & 1,153 \\
\hline 111 & 10000 & $(1,3)$ & 5 & 0,941 & 1,153 \\
\hline 112 & 10000 & $(1,3)$ & 10 & 0,941 & 1,153 \\
\hline 113 & 10000 & $(1,3)$ & 15 & 0,941 & 1,153 \\
\hline 114 & 10000 & $(1,3)$ & 20 & 0,942 & 1,153 \\
\hline 115 & 10000 & $(1,4)$ & 0 & 0,943 & 1,155 \\
\hline 116 & 10000 & $(1,4)$ & 2 & 0,945 & 1,158 \\
\hline 117 & 10000 & $(1,4)$ & 5 & 0,945 & 1,158 \\
\hline 118 & 10000 & $(1,4)$ & 10 & 0,945 & 1,158 \\
\hline 119 & 10000 & $(1,4)$ & 15 & 0,946 & 1,158 \\
\hline 120 & 10000 & $(1,4)$ & 20 & 0,945 & 1,158 \\
\hline 121 & 15000 & $(1,1)$ & 0 & 0,928 & 1,136 \\
\hline 122 & 15000 & $(1,1)$ & 2 & 0,93 & 1,139 \\
\hline 123 & 15000 & $(1,1)$ & 5 & 0,929 & 1,138 \\
\hline 124 & 15000 & $(1,1)$ & 10 & 0,929 & 1,138 \\
\hline 125 & 15000 & $(1,1)$ & 15 & 0,929 & 1,138 \\
\hline 126 & 15000 & $(1,1)$ & 20 & 0,929 & 1,138 \\
\hline 127 & 15000 & $(1,2)$ & 0 & 0,936 & 1,146 \\
\hline 128 & 15000 & $(1,2)$ & 2 & 0,938 & 1,149 \\
\hline 129 & 15000 & $(1,2)$ & 5 & 0,938 & 1,149 \\
\hline
\end{tabular}




\begin{tabular}{|c|c|c|c|c|c|}
\hline 130 & 15000 & $(1,2)$ & 10 & 0,938 & 1,149 \\
\hline 131 & 15000 & $(1,2)$ & 15 & 0,938 & 1,148 \\
\hline 132 & 15000 & $(1,2)$ & 20 & 0,938 & 1,149 \\
\hline 133 & 15000 & $(1,3)$ & 0 & 0,94 & 1,151 \\
\hline 134 & 15000 & $(1,3)$ & 2 & 0,942 & 1,154 \\
\hline 135 & 15000 & $(1,3)$ & 5 & 0,942 & 1,154 \\
\hline 136 & 15000 & $(1,3)$ & 10 & 0,942 & 1,154 \\
\hline 137 & 15000 & $(1,3)$ & 15 & 0,942 & 1,153 \\
\hline 138 & 15000 & $(1,3)$ & 20 & 0,942 & 1,154 \\
\hline 139 & 15000 & $(1,4)$ & 0 & 0,943 & 1,155 \\
\hline 140 & 15000 & $(1,4)$ & 2 & 0,945 & 1,157 \\
\hline 141 & 15000 & $(1,4)$ & 5 & 0,945 & 1,157 \\
\hline 142 & 15000 & $(1,4)$ & 10 & 0,945 & 1,158 \\
\hline 143 & 15000 & $(1,4)$ & 15 & 0,945 & 1,157 \\
\hline 144 & 15000 & $(1,4)$ & 20 & 0,945 & 1,158 \\
\hline 145 & 20000 & $(1,1)$ & 0 & 0,934 & 1,143 \\
\hline 146 & 20000 & $(1,1)$ & 2 & 0,931 & 1,14 \\
\hline 147 & 20000 & $(1,1)$ & 5 & 0,931 & 1,14 \\
\hline 148 & 20000 & $(1,1)$ & 10 & 0,931 & 1,14 \\
\hline 149 & 20000 & $(1,1)$ & 15 & 0,931 & 1,14 \\
\hline 150 & 20000 & $(1,1)$ & 20 & 0,93 & 1,14 \\
\hline 151 & 20000 & $(1,2)$ & 0 & 0,94 & 1,151 \\
\hline 152 & 20000 & $(1,2)$ & 2 & 0,939 & 1,15 \\
\hline 153 & 20000 & $(1,2)$ & 5 & 0,939 & 1,15 \\
\hline 154 & 20000 & $(1,2)$ & 10 & 0,94 & 1,151 \\
\hline 155 & 20000 & $(1,2)$ & 15 & 0,939 & 1,15 \\
\hline 156 & 20000 & $(1,2)$ & 20 & 0,939 & 1,15 \\
\hline 157 & 20000 & $(1,3)$ & 0 & 0,943 & 1,154 \\
\hline 158 & 20000 & $(1,3)$ & 2 & 0,943 & 1,155 \\
\hline 159 & 20000 & $(1,3)$ & 5 & 0,943 & 1,155 \\
\hline 160 & 20000 & $(1,3)$ & 10 & 0,943 & 1,155 \\
\hline 161 & 20000 & $(1,3)$ & 15 & 0,943 & 1,155 \\
\hline 162 & 20000 & $(1,3)$ & 20 & 0,943 & 1,155 \\
\hline 163 & 20000 & $(1,4)$ & 0 & 0,945 & 1,158 \\
\hline 164 & 20000 & $(1,4)$ & 2 & 0,946 & 1,159 \\
\hline 165 & 20000 & $(1,4)$ & 5 & 0,946 & 1,159 \\
\hline 166 & 20000 & $(1,4)$ & 10 & 0,946 & 1,159 \\
\hline 167 & 20000 & $(1,4)$ & 15 & 0,946 & 1,159 \\
\hline 168 & 20000 & $(1,4)$ & 20 & 0,946 & 1,159 \\
\hline 169 & 25000 & $(1,1)$ & 0 & 0,933 & 1,143 \\
\hline 170 & 25000 & $(1,1)$ & 2 & 0,932 & 1,142 \\
\hline 171 & 25000 & $(1,1)$ & 5 & 0,932 & 1,141 \\
\hline 172 & 25000 & $(1,1)$ & 10 & 0,932 & 1,141 \\
\hline 173 & 25000 & $(1,1)$ & 15 & 0,931 & 1,141 \\
\hline 174 & 25000 & $(1,1)$ & 20 & 0,932 & 1,141 \\
\hline
\end{tabular}




\begin{tabular}{|c|c|c|c|c|c|}
\hline 175 & 25000 & $(1,2)$ & 0 & 0,94 & 1,151 \\
\hline 176 & 25000 & $(1,2)$ & 2 & 0,939 & 1,15 \\
\hline 177 & 25000 & $(1,2)$ & 5 & 0,938 & 1,149 \\
\hline 178 & 25000 & $(1,2)$ & 10 & 0,939 & 1,15 \\
\hline 179 & 25000 & $(1,2)$ & 15 & 0,939 & 1,15 \\
\hline 180 & 25000 & $(1,2)$ & 20 & 0,939 & 1,15 \\
\hline 181 & 25000 & $(1,3)$ & 0 & 0,943 & 1,154 \\
\hline 182 & 25000 & $(1,3)$ & 2 & 0,944 & 1,156 \\
\hline 183 & 25000 & $(1,3)$ & 5 & 0,943 & 1,155 \\
\hline 184 & 25000 & $(1,3)$ & 10 & 0,943 & 1,155 \\
\hline 185 & 25000 & $(1,3)$ & 15 & 0,943 & 1,155 \\
\hline 186 & 25000 & $(1,3)$ & 20 & 0,943 & 1,155 \\
\hline 187 & 25000 & $(1,4)$ & 0 & 0,946 & 1,158 \\
\hline 188 & 25000 & $(1,4)$ & 2 & 0,947 & 1,16 \\
\hline 189 & 25000 & $(1,4)$ & 5 & 0,946 & 1,159 \\
\hline 190 & 25000 & $(1,4)$ & 10 & 0,947 & 1,16 \\
\hline 191 & 25000 & $(1,4)$ & 15 & 0,946 & 1,159 \\
\hline 192 & 25000 & $(1,4)$ & 20 & 0,946 & 1,159 \\
\hline 193 & 45000 & $(1,1)$ & 0 & 0,93 & 1,139 \\
\hline 194 & 45000 & $(1,1)$ & 2 & 0,926 & 1,134 \\
\hline 195 & 45000 & $(1,1)$ & 5 & 0,927 & 1,135 \\
\hline 196 & 45000 & $(1,1)$ & 10 & 0,927 & 1,135 \\
\hline 197 & 45000 & $(1,1)$ & 15 & 0,927 & 1,135 \\
\hline 198 & 45000 & $(1,1)$ & 20 & 0,927 & 1,135 \\
\hline 199 & 45000 & $(1,2)$ & 0 & 0,938 & 1,148 \\
\hline 200 & 45000 & $(1,2)$ & 2 & 0,936 & 1,146 \\
\hline 201 & 45000 & $(1,2)$ & 5 & 0,937 & 1,148 \\
\hline 202 & 45000 & $(1,2)$ & 10 & 0,937 & 1,147 \\
\hline 203 & 45000 & $(1,2)$ & 15 & 0,937 & 1,147 \\
\hline 204 & 45000 & $(1,2)$ & 20 & 0,937 & 1,147 \\
\hline 205 & 45000 & $(1,3)$ & 0 & 0,941 & 1,153 \\
\hline 206 & 45000 & $(1,3)$ & 2 & 0,941 & 1,153 \\
\hline 207 & 45000 & $(1,3)$ & 5 & 0,941 & 1,153 \\
\hline 208 & 45000 & $(1,3)$ & 10 & 0,941 & 1,153 \\
\hline 209 & 45000 & $(1,3)$ & 15 & 0,941 & 1,153 \\
\hline 210 & 45000 & $(1,3)$ & 20 & 0,941 & 1,153 \\
\hline 211 & 45000 & $(1,4)$ & 0 & 0,943 & 1,155 \\
\hline 212 & 45000 & $(1,4)$ & 2 & 0,944 & 1,156 \\
\hline 213 & 45000 & $(1,4)$ & 5 & 0,944 & 1,157 \\
\hline 214 & 45000 & $(1,4)$ & 10 & 0,945 & 1,157 \\
\hline 215 & 45000 & $(1,4)$ & 15 & 0,945 & 1,157 \\
\hline 216 & 45000 & $(1,4)$ & 20 & 0,944 & 1,157 \\
\hline 217 & 65000 & $(1,1)$ & 0 & 0,93 & 1,139 \\
\hline 218 & 65000 & $(1,1)$ & 2 & 0,927 & 1,135 \\
\hline 219 & 65000 & $(1,1)$ & 5 & 0,927 & 1,135 \\
\hline
\end{tabular}




\begin{tabular}{r|r|r|r|r|r|}
\hline 220 & 65000 & $(1,1)$ & 10 & 0,927 & 1,135 \\
\hline 221 & 65000 & $(1,1)$ & 15 & 0,927 & 1,135 \\
\hline 222 & 65000 & $(1,1)$ & 20 & 0,927 & 1,136 \\
\hline 223 & 65000 & $(1,2)$ & 0 & 0,938 & 1,148 \\
\hline 224 & 65000 & $(1,2)$ & 2 & 0,938 & 1,148 \\
\hline 225 & 65000 & $(1,2)$ & 5 & 0,938 & 1,149 \\
\hline 226 & 65000 & $(1,2)$ & 10 & 0,937 & 1,148 \\
\hline 227 & 65000 & $(1,2)$ & 15 & 0,937 & 1,148 \\
\hline 228 & 65000 & $(1,2)$ & 20 & 0,938 & 1,148 \\
\hline 229 & 65000 & $(1,3)$ & 0 & 0,941 & 1,152 \\
\hline 230 & 65000 & $(1,3)$ & 2 & 0,941 & 1,152 \\
\hline 231 & 65000 & $(1,3)$ & 5 & 0,941 & 1,153 \\
\hline 232 & 65000 & $(1,3)$ & 10 & 0,941 & 1,152 \\
\hline 233 & 65000 & $(1,3)$ & 15 & 0,941 & 1,152 \\
\hline 234 & 65000 & $(1,3)$ & 20 & 0,941 & 1,153 \\
\hline 235 & 65000 & $(1,4)$ & 0 & 0,943 & 1,154 \\
\hline 236 & 65000 & $(1,4)$ & 2 & 0,943 & 1,155 \\
\hline 237 & 65000 & $(1,4)$ & 5 & 0,943 & 1,155 \\
\hline 238 & 65000 & $(1,4)$ & 10 & 0,943 & 1,155 \\
\hline 239 & 65000 & $(1,4)$ & 15 & 0,943 & 1,155 \\
\hline 240 & 65000 & $(1,4)$ & 20 & 0,943 & 1,155 \\
\hline
\end{tabular}




\section{References}

[1] P. Sitikhu, K. Pahi, P. Thapa, and S. Shakya, "A Comparison of Semantic Similarity Methods for Maximum Human Interpretability”, vol. 1, 2019, pp. 1-4. DoI: 10.1109/AITB48515.2019.8947433.

[2] C. D. Manning, P. Raghavan, and H. Schütze, Introduction to Information Retrieval. USA: Cambridge University Press, 2008, ISBN: 0521865719.

[3] C. De Boom, S. Van Canneyt, T. Demeester, and B. Dhoedt, "Representation Learning for Very Short Texts Using Weighted Word Embedding Aggregation”, Pattern Recogn. Lett., vol. 80, no. C, pp. 150-156, Sep. 2016, ISSN: 0167-8655. DOI: 10.1016/j.patrec.2016.06.012.

[4] G. Kim and K. Cho, "Length-Adaptive Transformer: Train Once with Length Drop, Use Anytime with Search", ArXiv, vol. abs/2010.07003, 2020.

[5] O. Zafrir, G. Boudoukh, P. Izsak, and M. Wasserblat, "Q8BERT: Quantized 8Bit BERT”, ArXiv, vol. abs/1910.06188, 2019.

[6] H. Gong, Y. Shen, D. Yu, J. Chen, and D. Yu, "Recurrent Chunking Mechanisms for Long-Text Machine Reading Comprehension”, in Proceedings of the 58th Annual Meeting of the Association for Computational Linguistics, Association for Computational Linguistics, Jul. 2020, pp. 6751-6761. DOI: 10.18653/v1/2020.acl-main.603. [Online]. Available: https://www.aclweb.org/anthology/2020.aclmain.603.

[7] Y. Qi, D. Sachan, M. Felix, S. Padmanabhan, and G. Neubig, "When and Why Are Pre-Trained Word Embeddings Useful for Neural Machine Translation?", in Proceedings of the 2018 Conference of the North American Chapter of the Association for Computational Linguistics: Human Language Technologies, Volume 2 (Short Papers), New Orleans, Louisiana: Association for Computational Linguistics, Jun. 2018, pp. 529-535. Dor: 10.18653/v1/N18-2084. [Online]. Available: https://www.aclweb.org/anthology/N182084.

[8] D. Shen, G. Wang, W. Wang, M. R. Min, Q. Su, Y. Zhang, C. Li, R. Henao, and L. Carin, "Baseline Needs More Love: On Simple Word-Embedding-Based Models and Associated Pooling Mechanisms", in Proceedings of the 56th Annual Meeting of the Association for Computational Linguistics (Volume 1: Long Papers), Melbourne, Australia: Association for Computational Linguistics, Jul. 2018, pp. 440-450. DOI: 10.18653/v1/P18-1041. [Online]. Available: https://www.aclweb.org/anthology/P18-1041.

[9] A. Rücklé, S. Eger, M. Peyrard, and I. Gurevych, "Concatenated p-mean Word Embeddings as Universal Cross-Lingual Sentence Representations”, ArXiv, vol. abs/1803.01400, 2018.

[10] P. Turney and P. Pantel, "From Frequency to Meaning: Vector Space Models of Semantics", fournal of Artificial Intelligence Research, vol. 37, pp. 141-188, Mar. 2010. DOI: 10.1613/jair.2934.

[11] A. L. O. Shahmirzadi and K. Younge, "Text Similarity in Vector Space Models: A Comparative Study", in 2019 18th IEEE International Conference On Machine Learning And Applications (ICMLA), 2019, pp. 659-666. DOI: 10.1109/ICMLA.2019.00120.

[12] V. Gupta, A. Kumar, P. Nokhiz, H. Gupta, and P. Talukdar, "Improving Document Classification with Multi-Sense Embeddings", in 24th European Conference on Artificial Intelligence - ECAI 2020, Nov. 2020, pp. 2030-2037. DoI: 10.3233/FAIA200324.

[13] V. Mekala Dheeraj and Gupta, B. Paranjape, and H. Karnick, "SCDV : Sparse Composite Document Vectors using soft clustering over distributional representations", in Proceedings of the 2017 Conference on Empirical Methods in Natural Language Processing, Copenhagen, Denmark: Association for Computational Linguistics, Sep. 2017, pp. 659-669. Dor: 10.18653/v1/D17-1069. [Online]. Available: https://www.aclweb.org/anthology/D17-1069. 
[14] V. Gupta, H. Karnick, A. Bansal, and P. Jhala, "Product Classification in E-Commerce using Distributional Semantics", in Proceedings of COLING 2016, the 26th International Conference on Computational Linguistics: Technical Papers, Osaka, Japan: The COLING 2016 Organizing Committee, Dec. 2016, pp. 536-546. [Online]. Available: https://www.aclweb.org/anthology/C16-1052. 